Saúde e Pesquisa, Maringá (PR)

DOI: http://dx.doi.org/10.177651/1983-1870.2017v10n3p405-415

\title{
APLICABILIDADE DE UMA BATERIA NEUROPSICOLÓGICA EM IDOSOS COM COMPROMETIMENTO COGNITIVO LEVE E DOENÇA DE ALZHEIMER
}

\section{Mariana Bonotto Mallmann}

Mestre em Envelhecimento Humano, pela Universidade de Passo Fundo (UPF), Brasil. Doutorado em andamento em Psicologia na Universidade Católica Dom Bosco (UCDB), Brasil.

E-mail: marianamallmann@yahoo.com.br

\section{Marlene Doring}

Docente e orientadora do Programa de Pós-graduação em Envelhecimento Humano (Stricto sensu) da Universidade de Passo Fundo (UPF); Doutora em Saúde Pública, pela Universidade de São Paulo (USP), Brasil.
RESUMO: Analisar o desempenho de idosos com diagnóstico de Comprometimento Cognitivo Leve, com doença de Alzheimer (DA) em fase inicial e de idosos saudáveis, em uma bateria específica de testes neuropsicológicos. $\mathrm{Na}$ análise estatística, consideraram-se como significativos os testes com valor de probabilidade $<0,05$. Os resultados obtidos demonstraram que os testes neuropsicológicos pertencentes à bateria foram capazes de discriminar déficits cognitivos nas populações estudadas. Observou-se associação estatisticamente significativa entre todos os testes, quando realizada a comparação entre os grupos e o diagnóstico clínico. Os pacientes com DA apresentaram maior declínio em todos os testes quando em comparação com o grupo com Comprometimento Cognitivo Leve (CCL) e saudáveis. Assim, o estudo contribuiu para evidenciar os déficits cognitivos em idosos com comprometimento cognitivo leve e doença de Alzheimer, estabelecendo alguns padrões neuropsicológicos, o que auxiliará na realização de avaliações e diagnósticos efetivos, além de direcionar medidas interdisciplinares de intervenção mais apropriadas para essas populações.

PALAVRAS-CHAVE: Envelhecimento; Déficit; Avaliação; Demência.

\section{APPLICABILITY OF NEUROPSYCHOLOGICAL TESTS IN ELDERLY PEOPLE WITH MILD COGNITIVE IMPAIRMENT AND ALZHEIMER DISEASE}

\begin{abstract}
Current paper analyzes the performance of elderly people diagnosed with Mild Cognitive Impairment and with early phase Alzheimer's Disease and healthy elderly people, by a set of neuropsychological tests. Statistical Analysis tests were significant when probability was $<0.05$. Results showed that the neuropsychological tests discriminated cognitive deficits in the studied populations. There was a significant statistical association between all tests (when groups were compared) and clinical diagnosis. Patients with $\mathrm{AD}$ had the great slide in all tests when compared with patients with mild cognitive impairment and healthy elders. Neuropsychological standards are established and they will be of great help towards assessments and diagnoses. They will be an asset for more appropriate interdisciplinary measures of intervention in these populations.
\end{abstract}

KEY WORDS: Getting old; Deficit; Evaluation; Dementia. 


\section{INTRODUÇão}

O declínio cognitivo é um dos cuidados de saúde mais preocupantes do século XXI (NAGAMATSU et al., 2013). Sabe-se que ele pode ocorrer no envelhecimento natural ou significar um quadro patológico. Dentre os quadros patológicos que apresentam declínio cognitivo, encontra-se a Demência na Doença de Alzheimer (DDA) provável, considerada como a mais prevalente entre os quadros neurodegenerativos na população idosa (60 80\%), segundo World Alzheimer Report (2016), atingindo cerca de 5 a 7\% da população mundial com mais de 60 anos (PRINCE et al., 2013). Um artigo publicado por Godinho (2012) demonstrou que a taxa de incidência da Doença de Alzheimer (DA) numa população do sul do Brasil foi 14,8 por 1000 pessoas-ano. Além de gerar enorme impacto na vida do paciente, a DA também causa prejuízos na vida dos cuidadores.

Acrescenta-se, ainda, que apenas 40 a $50 \%$ das pessoas, que têm algum tipo de demência, receberam um diagnóstico (WORLD ALZHEIMER REPORT, 2015). Um dos fatores para isso é a dificuldade em conseguir identificar esses quadros clinicamente. Estudos assinalam que frente a essa dificuldade, a Avaliação Neuropsicológica (AN) surge como uma significativa contribuição (MILGRAM et al., 2006; YASSUDA et al., 2010).

Além das demências, conhece-se outro quadro, no qual os indivíduos também apresentam declínio cognitivo, e denomina-se Comprometimento Cognitivo Leve (CCL), mas este quadro é de magnitude insuficiente para interferir nas atividades da vida diária (AVD) do indivíduo. Sabe-se que indivíduos com CCL tem maior probabilidade de desenvolver demência. Ele é considerado um estado intermediário entre o envelhecimento normal e a DA, e, quando diagnosticado precocemente, poderá auxiliar no não desenvolvimento de um quadro neurodegenerativo.

Alguns autores demonstraram que a taxa de conversão de CCL para a DA é de $10 \%$ a $15 \%$ ao ano, enquanto pessoas saudáveis apresentam taxa de $1 \%$ a $2 \%$ ao ano (BRUSCOLI; LOVESTONE, 2004; SOARES et al., 2013). Dados epidemiológicos trazem taxas de prevalência de CCL entre 14 e 18\% para os indivíduos com idade superior a 70 anos (DALPUBEL et al., 2016).
Relatórios brasileiros de um estudo de Godinho (2012) demonstraram que a incidência de CCL foi de 13,2 por 1.000 pessoas/ano, e ainda afirmaram que a trajetória do CCL para conversão para demência foi de $38 \%$.

A falta de consenso quanto aos testes neuropsicológicos necessários para avaliar determinada patologia, aliado à carência de padróes estabelecidos para os comprometimentos esperados e a inópia de testes neuropsicológicos com estudos sobre suas propriedades psicométricas voltados à população idosa brasileira (HAMDAN; HOLLVEG, 2008; NITRINI et. al., 2005; REPPOLD et al., 2015), instiga tanto a necessidade de estudos, quanto a busca por instrumentos que permitam auxiliar no diagnóstico e tratamento das patologias. Desse modo, objetivou-se analisar o desempenho de idosos saudáveis, com diagnóstico de CCL e DDA provável em estágio inicial, em uma bateria específica $(\mathrm{BN})$ de testes neuropsicológicos, auxiliando assim no estabelecimento de padrões neuropsicológicos para estes quadros.

\section{METODOLOGIA}

O estudo foi realizado em um serviço particular de neurologia e neurocirurgia, na cidade de Passo Fundo, Rio Grande do Sul (RS). Os pacientes foram encaminhados pelos médicos neurologistas e neurocirurgiões membros do serviço.

Participaram do estudo 77 indivíduos, pacientes e/ou pessoas que possuíam algum vínculo empregatício com o local onde se realizou o estudo, com idade de 60 anos ou mais, com diferentes anos de escolaridade, entre um ano e mais de nove anos de estudo formal (a educação formal foi avaliada em anos, descontando-se repetências). Além disso, participaram pessoas com diferentes estados civis (solteiro, casado, viúvo e separado) e de níveis socioeconômicos diversos, todos residentes no Estado do Rio Grande do Sul. O estudo foi constituído por três grupos:

1) Vinte e cinco idosos com diagnóstico de DDA provável em estágio inicial, segundo os critérios do NINCDS-ADRDA (MCKHANN et al., 2011) e que possuíram pontuação igual a 1 na Escala Clínica de Demência (CDR). 
2) Vinte e seis idosos com diagnóstico de CCL, que preencheram critérios diagnósticos preconizados pelo Instituto Nacional de Envelhecimento Americano (National Institute on Aging) e a Associação de Alzheimer (Alzheimer's Association), (ALBERT et al., 2011) e que possuíram CDR $=0,5$.

3) Vinte e seis indivíduos sem história de comprometimento cognitivo ou de qualquer doença neurológica ou psiquiátrica, e que possuem $\mathrm{CDR}=0$, que fizeram parte do grupo de idosos saudáveis.

Foram considerados critérios de inclusão para o estudo: a) indivíduos com 60 anos e mais, com pontuação inferior ao ponto de corte (5/6) para depressão maior na Escala de Depressão Geriátrica (GDS-15) (PARADELA; LOURENÇO; VERAS, 2005); b) indivíduos com CCL e DDA provável em estágio inicial c) com, no mínimo, um ano de estudo formal, participantes que não passaram pela educação formal, mas foram capazes de ler e escrever frases simples, foram classificados como tendo um ano de educação formal; d) que porventura apresentaram diabetes mellitus e/ou hipertensão arterial, mas controladas.

Foram considerados critérios de exclusão para a participação da pesquisa aqueles: a) idosos com histórico sugestivo de tratamento psiquiátrico (com exceção de transtornos depressivos e/ou ansiosos); b) histórico de abuso de álcool e/ou droga; c) morbidades como acidente vascular encefálico, traumatismo crânio encefálico, epilepsia, doença de Parkinson, deficiência intelectual; d) déficits sensoriais, motores e/ou cognitivos (por outras etiologias), que comprometam o desempenho nos testes; e) déficit de audição que possa comprometer a comunicação; f) incapacidade visual que possa interferir na execução dos testes;

Avaliou-se a presença de sintomas psicóticos e/ou outras doenças psiquiátricas e neurológicas, por meio da entrevista clínica. Especificamente em relação a sintomas depressivos, foi utilizada a Escala de Depressão Geriátrica - GDS (YESAVAGE et al., 1982). Para avaliar a ansiedade foi utilizada a escala Beck de Ansiedade. O Mini Exame de Estado Mental - MEEM (BRUCKI et al., 2003; FOLSTEIN et al., 1975;) foi aplicado, com valores ajustados para a escolaridade, com o objetivo de excluir pacientes com demência em estágio moderado ou grave, na triagem. Os pacientes foram também submetidos ao questionário de atividades funcionais de (PFEFFER et al., 1982), sendo incluídos os que obtiveram pontuação menor ou igual a 5 .

\subsection{PROCEDIMENTOS DE COLETA DE DADOS E INS- TRUMENTOS}

Posteriormente à obtenção da carta de autorização da instituição e da aprovação do projeto pelo Comitê de Ética em Pesquisa da Universidade de Passo Fundo (parecer 643.771/2014), deu-se início à coleta dos dados. A amostra de idosos foi recrutada em um centro especializado de neurologia e neurocirurgia, na cidade de Passo Fundo, RS.

Após o diagnóstico médico, os idosos foram encaminhados para triagem que foi realizada aplicandose testes psicológicos para verificar os critérios de inclusão à pesquisa. Os idosos que foram considerados elegíveis para a pesquisa foram encaminhados para a avaliação neuropsicológica, com hora e data marcadas individualmente. A bateria neuropsicológica foi construída de forma a ser aplicada integralmente, além da entrevista e rastreio cognitivo, em uma única sessão de, no máximo, 120 minutos.

Foram selecionados os seguintes testes para compor a BN: Instrumento de Neuropsicologia Breve; Teste de Trilhas partes A e B; Fluência Verbal Semântica e Fluência Verbal Fonêmica; Span de Dígitos, ordem direta e ordem inversa; Cubos de Corsi; Bateria de Avaliação Frontal; Token Test; Teste de Aprendizagem Auditivo Verbal de Rey.

Os participantes ou cuidadores que não mostraram interesse em ingressar no estudo foram atendidos normalmente, não sofrendo qualquer ônus ou penalidade com a não participação. Ressalta-se que os autores não possuem quaisquer conflitos de interesse relacionados a esse estudo.

\subsubsection{Análise dos dados}

As variáveis numéricas dos testes da BN foram expressas como média \pm desvio-padrão ou mediana (percentil 25 - percentil 75) conforme apresentaram 
distribuição normal ou não normal. As variáveis categóricas foram expressas como frequência absoluta e relativa. A normalidade foi avaliada pela análise visual dos histogramas e confirmada pelo teste de KolmorogovSmirmov.

As diferenças da distribuição das variáveis desses testes que compuseram a bateria neuropsicológica entre os grupos formados conforme as categorias definidas pela Associação Brasileira de Neurologia para o grupo com CCL e os critérios do NINCDS-ADRDA (MCKHANN et al., 2011) para o grupo com DA, foram avaliadas utilizandose análise de variância não paramétrica de KolmogorovSmirnov e, as comparações aos pares, utilizando-se o teste post hoc e do Dunn-Bonferroni. Consideraram-se como significativos os testes com valor de probabilidade $<0,05$.

\section{RESULTADOS}

Neste estudo foram avaliados 77 indivíduos, sendo 25 pacientes com DDA provável em estágio inicial, 26 pacientes com CCL e 26 pacientes considerados saudáveis.

A seguir, demonstram-se em tabelas as características sociodemográficas (Tabela 1), as características dos hábitos de vida (Tabela 2) e as condições de saúde (Tabela 3) da população do estudo.

Tabela 1. Características sociodemográficas da população do estudo $(n=77)$

\begin{tabular}{lccc}
\hline & $\begin{array}{c}\text { Demência na doença de } \\
\text { Alzheimer } \\
(\boldsymbol{n}=25)\end{array}$ & $\begin{array}{c}\text { Comprometimento cognitivo } \\
\text { leve } \\
(\boldsymbol{n}=26)\end{array}$ & $\begin{array}{c}\text { Saudáveis } \\
(\boldsymbol{n}=\mathbf{2 6})\end{array}$ \\
\hline Sexo feminino & $18(72,0 \%)$ & $16(61,5 \%)$ & $10(38,5 \%)$ \\
Idade & $75,8 \pm 5,5$ & $69,4 \pm 7,1$ & $67,4 \pm 6,3$ \\
\hline Estado civil & - & $4(15,4 \%)$ & $1(3,8 \%)$ \\
\hline \multicolumn{1}{c}{ Solteiro } & $17(68,0 \%)$ & $20(76,9 \%)$ & $29(73,1 \%)$ \\
Casado & $8(32,0 \%)$ & $2(7,7 \%)$ & $5(19,2 \%)$ \\
Viúvo & - & & $1(3,8 \%)$ \\
Divorciado & $3(12,0 \%)$ & $2(7,7 \%)$ & $8(30,8 \%)$ \\
\hline Classe econômica & $18(72,0 \%)$ & $14(53,9 \%)$ & $2(7,7 \%)$ \\
\hline A1 e A2 & $4(16,0 \%)$ & $10(38,5 \%)$ & $1(3,8 \%)$ \\
B1 e B2 & & $4(15,4 \%)$ & $19(73,1 \%)$ \\
C1 e C2 & - & $20(76,9 \%)$ & $5(23 \%)$ \\
\hline Escolaridade & $17(68,0 \%)$ & $2(7,7 \%)$ & \\
\hline 1 a 4 anos & $8(32,0 \%)$ & & \\
5 a 8 anos & & & \\
+ de 9 anos & & & \\
\hline
\end{tabular}

Valores expressam frequência absoluta e relativa ou média \pm desvio-padrão 
Tabela 2. Condições de saúde da população de estudo $(n=77)$

\begin{tabular}{lccc}
\hline & $\begin{array}{c}\text { Demência na doença de } \\
\text { Alzheimer } \\
(\boldsymbol{n}=\mathbf{2 5})\end{array}$ & $\begin{array}{c}\text { Comprometimento } \\
\text { cognitivo leve } \\
(\boldsymbol{n}=\mathbf{2 6})\end{array}$ & $\begin{array}{c}\text { Saudáveis } \\
(\boldsymbol{n}=\mathbf{2 6})\end{array}$ \\
\hline Tabagismo & - & - & $1(3,8 \%)$ \\
Consumo de álcool & - & $2(7,7 \%)$ & $12(46,2 \%)$ \\
Atividade física & $10(40,0 \%)$ & $15(57,7 \%)$ & $14(53,8 \%)$ \\
\hline Diagnósticos prévios & & & \\
\hline Ansiedade e/ou depressão & $13(52,0 \%)$ & $7(26,9 \%)$ & $8(30,8 \%)$ \\
$\quad$ Insônia & $10(40,0 \%)$ & $12(46,2 \%)$ & $10(38,5 \%)$ \\
\hline Medicação de uso crônico & & & \\
\hline \multicolumn{1}{|l}{ Antidepressivo } & $15(64,0 \%)$ & $8(34,6 \%)$ & $10(42,3 \%)$ \\
\hline
\end{tabular}

Valores expressam frequência absoluta e relativa ou média \pm desvio-padrão

$\mathrm{Na}$ Tabela 3 estão descritas as associações entre os diagnósticos clínicos e as variáveis da bateria de avaliação neuropsicológica $(\mathrm{n}=77)$.

Tabela 3. Comparação entre os diagnósticos clínicos e as variáveis da bateria de avaliação neuropsicológica $(\mathrm{n}=77)$

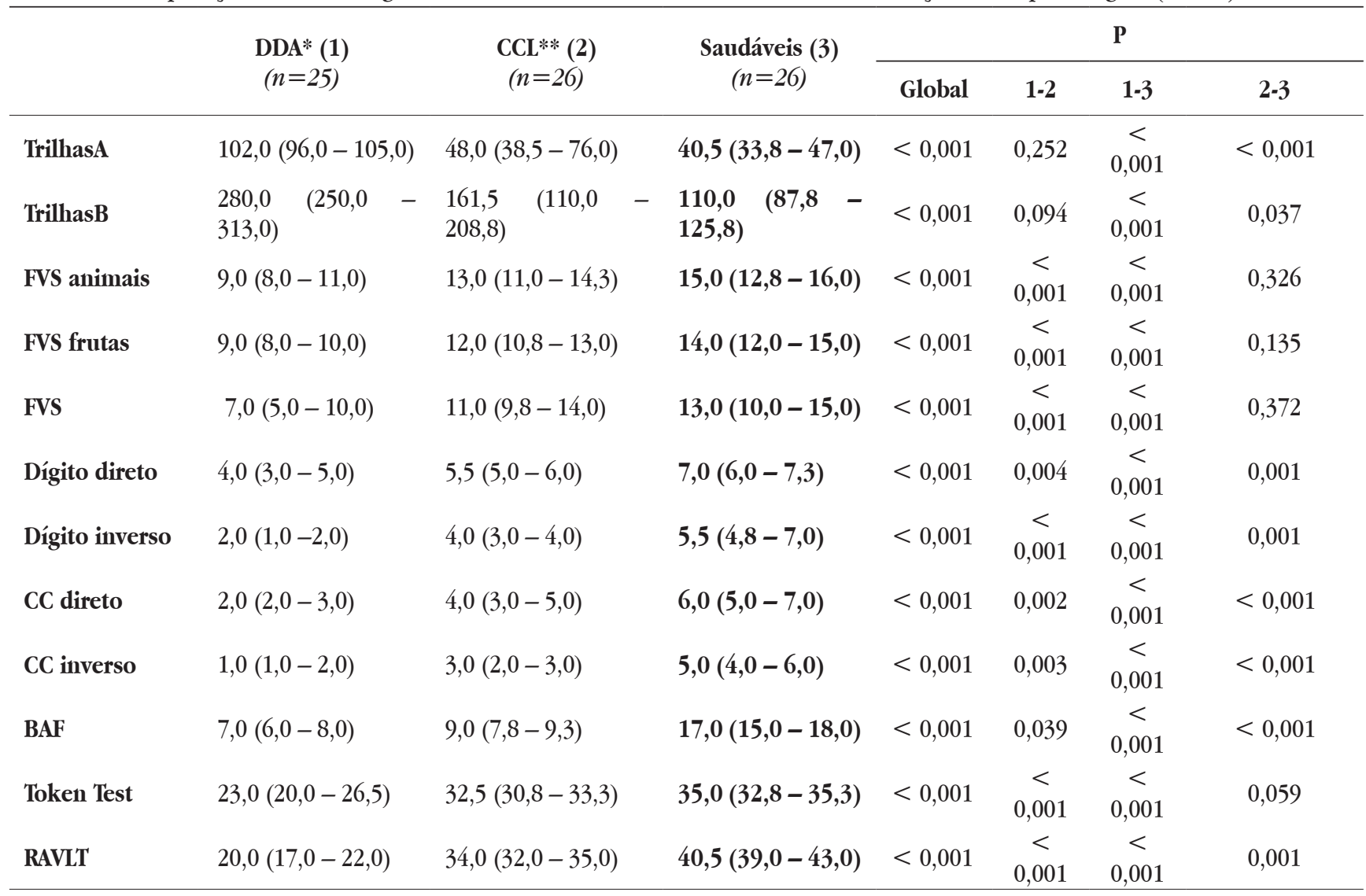

Valores expressam mediana (percentil25 - percentil75). * - Demência na doença de Alzheimer. ** - Comprometimento cognitivo leve. FVS - Fluência verbal semântica. CC - Cubos de Corsi. BAF - Bateria de avaliação frontal. RAVLT - Rey Auditory Verbal Learning Test Observou-se associação estatisticamente significativa entre todos os testes apresentados na Tabela 4 e o diagnóstico clínico. 
Os pacientes com DDA apresentaram maior declínio em todos os testes quando em comparação com o grupo com CCL e saudáveis.

Na Tabela 4 apresentam-se os valores encontrados nas tarefas do teste Neupsilin, que também pertenceu à bateria neuropsicológica, porém é um teste de uso restrito a psicólogos.

Tabela 5. Dados obtidos no teste Neupsilin

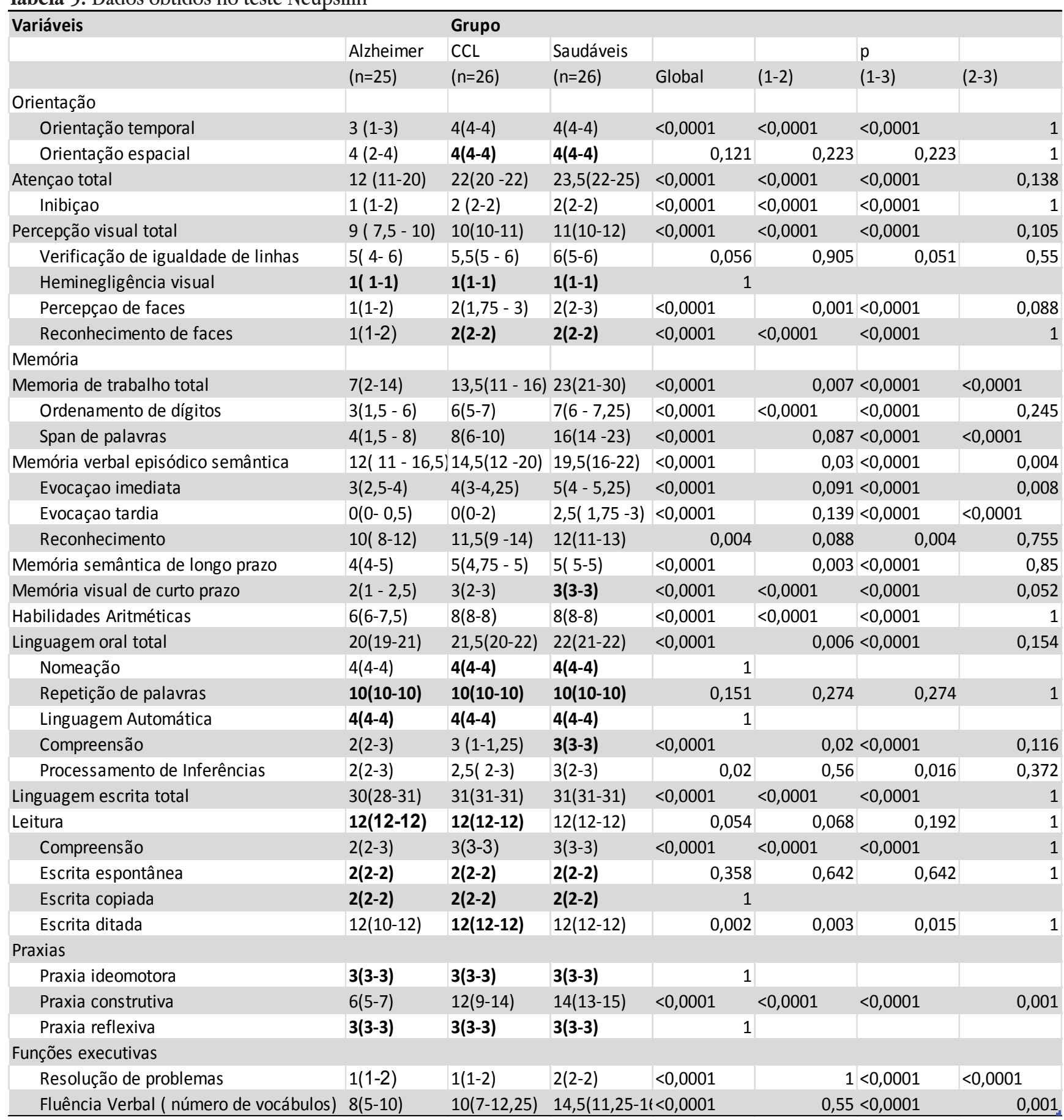

O post hoc análise identificou desempenho significativamente menor do grupo DDA, em comparação com o desempenho do grupo de saudáveis em pelo menos um dos subtestes de todos os domínios cognitivos avaliados. 


\section{DISCUSSÃO}

Alguns critérios foram necessários para a inclusão dos pacientes na amostra, entre eles a avaliação da presença de sintomas depressivos (GDS) e o desempenho nos escores do MEEM. A esse respeito, Mattos e Paixão (2010) afirmam que a presença de quadros depressivos é um desafio para uma avaliação neuropsicológica porque, a miúde, essas condições se associam a queixas cognitivas. Sabe-se, que a depressão pode trazer rebaixamento em diversas funções cognitivas.

O intuito deste procedimento foi, portanto, isolar a variável "depressão" como possível variável interveniente nos resultados dos testes, pois a depressão pode causar rebaixamentos em diversas funções cognitivas.

A análise estatística dos resultados mostrou que a comparação realizada entre os três grupos apresentou diferença significativa nas funções cognitivas avaliadas por meio da bateria de testes neuropsicológicos aplicada, o que representa um ponto essencial para a validade do trabalho. Mais especificamente, em todos os testes da bateria neuropsicológica, como em conformidade com os critérios já estabelecidos para essas populações, os pacientes com DDA apresentaram maior comprometimento nas funções avaliadas, quando em comparação com o grupo CCL e saudáveis.

Em comparação com o grupo de idosos saudáveis, quando os confrontou com o grupo de idosos com CCL, no teste de triagem Neupsilin, pertencente à bateria neuropsicológica aplicada, não se encontrou diferença significativa em diversas funções cognitivas avaliadas: orientação espacial e temporal, atenção, percepção visual, memória visual de curto prazo, memória verbal episódico semântica, linguagem oral e escrita. Em compensação, quando se comparou o grupo saudável com o grupo com DDA, apenas não se encontrou, diferença significativa na função cognitiva de orientação espacial.

Em conformidade a esses achados, algumas pesquisas indicam que o funcionamento executivo, atencional e alguns aspectos mnemônicos não estariam, a priori, tão prejudicados nos indivíduos com comprometimento cognitivo leve, pois estes ainda preservam sua AVDs; fato que na DA é considerado um dos critérios centrais para o diagnóstico (FROTA et al., 2011; MCKHANN et al., 2011; SPEARLING et al., 2011).
Ainda quando se comparou CCL com saudáveis, na avaliação da variável atenção, que foi realizada tanto no Neupsilin, quanto no teste de trilhas, não se encontrou diferença estatística, o que demonstra que a sessão do Neupsilin, que avalia atenção e o teste de trilhas, não foi capaz de discriminar essas populações.

Somando-se a isso, o teste de trilhas também não conseguiu diferenciar as populações DDA de CCL, assim, como a BAF e o subteste de fluência verbal do Neupsilin, nos achados desta pesquisa. Em contrapartida a esses achados, literaturas específicas demonstraram que o teste de trilhas é um teste neuropsicológico muito usado para identificar CCL e demência (GIL; BUSSE, 2009; CASANOVAet al., 2009).

$\mathrm{O}$ instrumento utilizado para mensurar a flexibilidade cognitiva, FAS, também não se mostrou adequado para os propósitos desta pesquisa. Ambos os testes não conseguiram diferenciar CCL de DDA, porém, a fluência verbal fonêmica do Neupsilin demonstrou diferença significativa nestas populações. Esses achados comprovam a importância da aplicação de testes de triagem com testes específicos para determinada função, ou seja, da utilização de uma bateria neuropsicológica.

Durante a escolha dos testes utilizados para a formação da bateria neuropsicológica, baseou-se no conhecimento de que determinados testes estariam sendo aplicados para avaliar prioritariamente determinada função. Entretanto, destaca-se que, embora os testes neuropsicológicos fossem desenvolvidos a partir de um paradigma que envolva uma determinada função, de modo geral esses testes não avaliam uma única função isoladamente, até mesmo porque exigem a integração dos diferentes sistemas neuronais para serem realizados (BOLFER, 2009). O exemplo disso percebe-se nos testes de trilhas parte A e B. Alguns padrões neuropsicológicos já foram estabelecidos para as populações clínicas estudadas (CCL e DDA) em alguns estudos anteriores, como o de Gagnon e Belleville (2011), que observaram que desde o início da DA, a memória de trabalho e o processo atencional estão alterados.

Outros estudos têm demonstrado que as primeiras manifestações da DA é o declínio cognitivo na memória episódica e nas habilidades visuoespacias (BURNS， 2009; KUZMICKIENÉ, 2016; ALZHEIMER 
ASSOCIATION, 2016). Déficits de memória de trabalho e funções executivas são frequentemente observados em pacientes com CCL (ALBERT et al., 2001, HUNTLEYAND HOWARD, 2010). Uma dificuldade na inibição também foi observada nesses pacientes (LONIE et al, 2009; SINAI et al. 2010; CLÉMENT et al., 2012; AURTENETXE, 2016). Alguns estudos sugerem a ocorrência de alterações significativas na memória de trabalho tanto nos quadros de CCL, quanto de DA (PETERSEN et al., 2001; PETERSEN, 2004; MALLOY-DINIZ et al., 2010; ALBERT et al., 2011; FROTA et al., 2011; BADDELEY; ANDERSON; EYSENKE, 2011).

Por esses estudos anteriores e a demonstração de que a memória pode estar prejudicada tanto na DA quanto no CCL, optou-se, nesta pesquisa, por um teste de memória verbal contemplando a curva de aprendizagem, a evocação tardia, e o reconhecimento, o RAVLT, justamente pelos estudos neuropsicológicos indicarem ser este o tipo de teste mais sensível para a detecção da DA (MALLOYDINIZ et al., 2007). Em conformidade a isso, as análises realizadas neste estudo, apontaram para significância estatística entre os grupos em todas as etapas do teste quando se compararam os três grupos, indicando ser este realmente um teste no qual se diferencia envelhecimento saudável do patológico.

Ademais, sabe-se que os testes de memória que requerem aprendizado e resgate de palavras, como é o caso do RAVLT, se mostram mais perceptíveis aos déficits da memória episódica para discriminação nos casos do CCL ou da DA (HAMDAM, 2010; CAIXETA FERREIRA, 2012; MIOTTO et al., 2014), o que está em concordância também com os achados deste estudo.

Ainda em relação à habilidade de memória de trabalho, que também foi avaliada por meio dos testes Span de dígitos e cubos de Corsi, os resultados encontrados também foram capazes de apontar padrões que distinguissem os três grupos. Segundo estudos anteriores (MILNER, 1971; GROSSI; BECKER; TROJANO, 1994) no teste de cubos de Corsi, os resultados sofrem interferência da idade, sexo e escolaridade, o que não foi considerado nesse estudo.

Quanto ao perfil neuropsicológico do que se espera no envelhecimento normal não é incomum haver disfunção executiva, o que, muitas vezes, dificulta na identificação se o déficit é produto de um processo fisiológico ou de um patológico (CAIXETA et al., 2014), pois, o mesmo também pode ocorrer tanto no CCL, como na DA, como comprovaram os achados do nosso estudo. Grady (2008) demonstrou que o declínio cognitivo associado ao envelhecimento saudável se inicia com as memórias episódicas. Ainda, Miotto (2012) sugere que as alterações cognitivas com o envelhecimento estão associadas, entre outras funções, à memória de trabalho.

A praxia ideomotora não demonstrou alteração em nenhuma das populações estudadas, porém encontrou-se déficit considerado moderado na praxia construtiva em pacientes com DA em fase inicial. Contrariamente aos nossos achados, um estudo de revisão de literatura de 2012 demonstrou que a praxia ideomotora foi considerada a mais frequente na DA desde o estágio inicial da doença (LIMA et al., 2012). Outros estudos, que estão em harmonia com os nossos resultados, afirmam que o declínio na linguagem e na praxia são normalmente mais evidentes em estágios moderados e severos da doença (LOPEZ et al., 2008; LAM et al., 2013;).

Em relação às funções executivas, alguns subcomponentes demonstraram-se rebaixados na DDA quando em comparação com os outros grupos. Estudos descrevem que a deterioração da cognição na DA já está mais avançada, sobretudo nas áreas cerebrais terciárias, ou seja, nas regiões responsáveis pelo funcionamento executivo (MALLOY-DINIZ et al., 2008; ALBERT et al., 2011; GLENN-MATTFIN, 2011; GRANDE，2013). Outras pesquisas indicam que pessoas que apresentam disfunções executivas têm maior impacto nas AVD e na qualidade de vida do que as disfunções na memória (VIDONI et al., 2011).

Algumas limitações metodológicas devem ser destacadas para analisar corretamente os resultados desta pesquisa: a) os dados coletados foram extraídos de amostras de conveniência de um consultório particular, onde a maioria das pessoas tinha nível socioeconômico alto; b) a amostra não é homogênea (n de pacientes aleatórios não pareados, classes sociais distintas, escolaridade, gênero) explicitando desequilíbrio na análise de alguns dados. 


\section{CONCLUSÃO}

A Bateria Neuropsicológica (BN) demonstrou capacidade de identificar déficits cognitivos nas populações estudadas nas funções cognitivas avaliadas. Além disso, o estudo mostrou diferenças significativas entre os três grupos CCL, DDA e os saudáveis.

Todos os testes pertencentes a BN demonstraram serem capaz de discriminar déficits cognitivos nas populações estudadas.

O diagnóstico baseado na avaliação neuropsicológica demonstrou associação com o diagnóstico realizado pela avaliação médica nos três grupos estudados.

Sugere-se, para futuro estudo, aumento da amostra e validação de todos os testes que fazem parte da bateria neuropsicológica.

\section{REFERÊNCIAS}

ALBERT, M. et al. The Diagnosis of Mild Cognitive ImpairmentduetoAlzheimer'sDisease: Recommendations from the National Institute on Aging-Alzheimer's Association Workgroups on Diagnostic Guidelines for Alzheimer's Disease. Alzheimer's \& dementia : the journal of the Alzheimer's Association, p. 270-279, 2011. < <ttp://doi.org/10.1016/j.jalz.2011.03.008>. Acesso em: 15 maio 2016.

ALZHEIMER'S ASSOCIATION. 2009. Disponível em: $\quad<$ https://www.alz.co.uk/research/files/ WorldAlzheimerReport.pdf $>$. Acesso em: 15 maio 2016.

ALZHEIMER'S ASSOCIATION. 2015. The global impact of dementia. Disponível em: <https://www.alz.co.uk/ research/world-report-2015> . Acesso em: 15 maio 2016.

ASSOCIATION WORKING GROUP. Alzheimer's and Dementia, v. 7, n. 3, p. 270-27, 2011. Disponível em: $<$ http://www.alz.org/documents_custom/diagnositic_ recommendations_mci_due_to_alz_proof.pdf $>$. Acesso em: 10 maio 2016.

AURTENETXE, S. et al. Interference Impacts Working Memory in Mild Cognitive Impairment. Frontiers in Neuroscience, v.10, p. 443. 2016. Disponível em:
< http://doi.org/10.3389/fnins.2016.00443> $>$. Acesso em: 15 maio 2016.

AZEVEDO, P. H. Avaliação neuropsicológica das funções executivas em com comprometimento cognitivo leve e demência do tipo Alzheimer: um estudo comparativo. 2013. Dissertação (Mestrado em Psicologia) - Programa de Pós-Graduação em Psicologia, na Linha de Pesquisa: Avaliação e Reabilitação Neuropsicológica, Universidade Federal do Paraná. Curitiba, Paraná. Disponível em: < http://www.humanas. ufpr.br/portal/psicologiamestrado/files/2012/05/PauloHenrique-Grande-Dissetacao1.pdf $>$. Acesso em: mai./ jun. 2016.

BATTISTONI, S. S. T.; NERI, A. L.; CUPERTINO, A. P. F. B. Validade da escala de depressão do Center for Epidemiological Studies entre idosos brasileiros. Rev. Saúde Pública [online], v.41, n.4, p. 598-605, 2007.

BADDELEY, A. ANDERSON, M. C., EYSENCK, M. W. Memória. Porto Alegre: Artmed, 2011.

BOLFER, C. Avaliação neuropsicológica das funções executivas e da atenção em crianças com transtorno do déficit de atenção/hiperatividade (TDAH). 2009. Dissertação (Mestrado em Ciências, área de concentração neurologia) - Faculdade de Medicina, Universidade de São Paulo.

BRUSCOLI, M.; LOVESTONE, S. I. MCI really just early dementia? A systematic review of conversion studies. International Psychogeriatrics, v. 16, n. 2, p. 129-140, 2004.

BURNS, A. ILIFFE, S. Dementia. Journal BMJ,.v. 338, 2009. DOI: https://doi.org/10.1136/bmj.b75.

CAIXETA, R. C. A. Associação entre Papilomavírus Humano, Vaginose Bacteriana e Inflamação Cervical e a detecção de anormalidades no exame citológico de adolescentes e mulheres jovens. 2014. Dissertação (Mestrado em Medicina Tropical e Saúde Pública com área de concentração em Microbiologia), Universidade Federal de Goiás, Goiânia.

CASANOVA P. S.; CASANOVA P. C.; CASANOVA C. C. Deterioro cognitivo em La terceira edad.Cubana Med Gen Int. v. 20, p. 5-6, 2004. 
COUTINHO G.; OLIVEIRA R.; MOLL J., et al. Is it possible to identify individuals with mild cognitive impairment and Alzheimer's disease using a 30-minute neuropsychologic al battery? Rev Psiq Clin., v. 40, n. 4, p. 135-143, 2013.

DALPUBEL, D. et al. Sintomas depressivos no comprometimento cognitivo leve: revisão sistemática. Revista Hospital Universitário Pedro Ernesto, v.15, n.1, p. 20-27, 2016.

DALPUBEL, D. Propriedades psicométricas de um protocolo neuropsicológico breve para uso em populações geriátricas. Rev Psiq Clín., v.37, n. 6, p. 24650,2010 .

DE PAULA, et al. Screening for executive dysfunction with the frontal assessment battery: psychometric properties analysis and representative normative data for brazilian older adults. Psicol. pesq., Juiz de Fora, v. 7, n. 1, p. 89-98, jun. 2013.

FROTA, N. A. F. et al. Critérios para o diagnóstico de doença de Alzheimer. In Dement Neuropsychol. V. 1, p. 5-1, 2011.

GAGNON, L. G.; BELLEVILLE, S. Working memory in mild cognitive impairment and Alzheimer's disease: Contribution of forgetting and predictive value of complex spantasks. Neuropsychology, v. 25, n. 2, p. 226-236, 2011.

GIL, G., BUSSE, A. L. Avaliação neuropsicológica e o diagnóstico de demência, comprometimento cognitivo leve e queixa de memória relacionada à idade. Arquivos Médicos dos Hospitais e da Faculdade de Ciências Medicas da Santa Casa de São Paulo, v. 54, n. 2, p. 44-50, 2009.

GLENN MATTFIN, C. M. P. Neuropsicologia das funções executivas. Fisiopatologia. Rio de janeiro. Ed. Guanabara, v. 8, 2011.

GODINHO C. C. Incidência de demência e comprometimento cognitivo leve e identificação de preditores numa amostra de base populacional. 2012. Tese. (Doutorado em Medicina) - Universidade Federal do Rio Grande do Sul, Porto Alegre.

GRADY, C. L., Cognitive Neuroscience of Aging. Annals of the New York Academy of Sciences, v. 1124, p. 127144. doi:10.1196/annals.1440.009, 2008.
GRANDE P. H. A. Avaliação neuropsicológica das funções executivas em idosos com comprometimento cognitivo leve e demência do tipo alzheimer: um estudo comparativo. 2013. Dissertação (Mestrado em Avaliação e Reabilitação Neuropsicológica) - Programa de Pós Graduação em Psicologia, Universidade Federal do Paraná, Curitiba.

GROSSI, D; BECKER, J. T.; TROJANO, L. Visuospatial imagery in Alzheimer`s disease. Percept and Mot Skills, v. $12, \mathrm{n} .4,1994$.

HAMDAN, A. C.; HOLLVEG, P. Avaliação Neuropsicológica em Idosos.RBCEH, Curitiba, v.5, n.2, p. 110-23, 2008.

HENRY J. D.; CRAWFORD J. R.; PHILLIPS L. H. Verbal fluency performance in dementia of the Alzheimer's type: a meta-analysis. Neuropsychology, v. 12, 2004.

HUNTLEY J. D.; HOWARD R. J. Workingmemory in earlyAlzheimer'sdisease: a neuropsychologicalreview. Int J Geriatric Psychiatry., v. 25, n. 2, p.121-32, 2010. DOI: 10.1002/gps.2314.

KUZMICKIENÉ, J.; KAUBRYS, G. Specific Features of Executive Dysfunction in Alzheimer-Type Mild Dementia Based on Computerized Cambridge Neuropsychological Test Automated Battery (CANTAB) Test Results. Medical Science Monitor: International Medical Journal of Experimental and Clinical Research, v.22, p. 3605-3613. Disponível em: <http://doi.org/10.12659/ MSM.900992> . Acesso: 10 maio 2016.

LAM, B. et al. Clinical, imaging and pathological heterogeneity of the Alzheimer's disease syndrome. Alzheimers Res Ther, v. 12, 2013.

LIMA, N. M. F. V; SERVELHERE, K. R.; MATOS A. R. O perfil das apraxias na doença de Alzheimer. Revista ensaios e Ciência Ciência Biológicas, Agrárias e da Saúde, v. 16, n 1, 2012.

LONIE J. A. et al., Dual task performance in early Alzheimer's disease, amnestic mild cognitive impairment and depression. Psychol. Med. v. 39, p. 23-31. 10.1017/ S0033291708003346

LOPEZ, O. L, DeKosky S. T. Clinical symptoms in Alzheimer's disease. In: Duyckaerts C, Litvan I, editors. Dementias. Handbook of Clinical Neurology, 2008. 
MALLOY-DINIZ, L. F. et al. Neuropsicologia das funções executivas. In: FUENTES, D.; MALLOY-DINIZ, L. F.; CAMARGO, C. H. P.; COSEnZA, R. M. (Ed.). Neuropsicologia: teoria e prática. Porto Alegre: Artmed, 2008.

MATTOS, P.; PAIXÃO, C. M., Avaliação Cognitiva de Idosos. Envelhecimento e Comprometimento Cognitivo Leve. [s.l.; s.n.], 2010.

MCKHANN, G. M. et al. The diagnosis of dementia due to Alzheimer's disease: recommendations from National Institute on Aging and the Alzheimer's Association workgroup. Alzheimer's and Dementia, Rockville, v.7, n.3, 2011.

MILGRAM, N. W. et al. Neuroprotective effects of cognitive enrichment. Canadá: University of Toronto, 2000 .

MILNER, B. Inter hemispheric diferences in the localization on psychological processes in man. Br Med Bull, 1971.

MIOTTO, E.C. et al. Neuropsicologia Clínica. São Paulo: Roca, 2012.

MORRIS J. C. Clinical Dementia Rating: a reliable and valid diagnostic and staging measure for Dementia of the Alzheimer type. Int Psychogeriatric v. 9, n. S1, p. S173-S176, 1997.

NAGAMATSU, L. et al. Physical Activity Improves Verbal and Spatial Memory in Older Adults with Probable Mild Cognitive Impairment: A 6-Month Randomized Controlled Trial. Journal of Aging Research; v. 2013. Disponível em: < http://dx.doi.org/10.1155/2013/861893>.

NITRINI, R. et al. Diagnóstico de doença de Alzheimer no Brasil: avaliação cognitiva e funcional. Recomendações do Departamento Científico de Neurologia Cognitiva e do Envelhecimento da Academia Brasileira de Neurologia. Arq. Neuropsiquiatr.; v. 63, n. 3A, 2005.

PETERSEN, R.C. et al. Practice parameter: early detection of dementia: mild cognitive impairment (an evidencebased review). Neurology, v. 56, n. 9, p. 1133-1142, 2001.
PETERSEN, R.C. Mild cognitive impairment as a diagnostic entily. Journal of Internal Medicine, v. 256, n. 3, p. 183194, 2004.

PETERSEN, R. C. Prevalence of mild cognitive impairment is higher in men: The MayofClinicStudyofAging. Neurology, 2010.

PRINCE, M. et al. The global prevalence of dementia: A systematic review and meta analysis. Alzheimer's \& Dementia: The Journal of the Alzheimer's Association, v. 9, n. 1, p. 63 - 75, 2013. doi: 10.1016/j. jalz.2012.11.007.

RADANOVIC M. et al. Contribuition to the evaluating of language disturbances in subcortical lesions. Arq. Neuropsiquiatr., v. 62, n. 1, p. 51-7, 2004.

REPPOLD, C. T. et al. Contribuições da psicometria para os estudos em neuropsicologia cognitiva. Psicologia: teoria pratica, São Paulo, v. 17, n. 2, p. 94-106, 2015.

SINAI M. et al., Tasks witching performance reveals heterogeneity amongst patients with mild cognitivei mpairment. Neuropsychology, v. 24, p. 757-774, 2010.

SPERLING, R. A. et al. Toward defining the preclinical stages of Alzheimer's disease: Recommendations from the National Institute on Aging-Alzheimer's Association work groups on diagnostic guide lines for Alzheimer's disease. Alzheimer's\&Dementia: The Journal of the Alzheimer's Association, v. 7, n. 3, p. 280-292, 2011.

VIDONI E. D. et al., Alzheimer diseasebiomarkers are associatedwithbodymass index. Neurology, v. 77, p. 1913-1920, 2011

WECHSLER, D. Escala de Inteligência Wechsler para Adultos, 1993. Adaptação Brasileira: Elizabeth do Nascimento. [s.1.]: Casa do Psicólogo, 2004.

Recebido em: 2017-05-30 Aceito em: 2017-08-23 